\title{
Formation control using optimal time multiplexing
}

\author{
Harsh Oza $^{1}$, Ravi Banavar ${ }^{1}$, Sukumar Srikant ${ }^{1}$, I.C. Morărescu ${ }^{2}$
}

\begin{abstract}
Lack of availability of adequate bandwidth for simultaneous communication between a large number of agents and the central processing unit motivates us to study a multiagent system employing time multiplexed control. Achieving a formation under such time-multiplexing constraints is posed as a discrete constrained optimal control problem. Employing the discrete Pontryagin Maximum Principle (DMP) we obtain first order necessary conditions to be satisfied by an optimal control law, which tackles both problems of formation control and time multiplexing simultaneously. The information structure of relative states is embedded into the problem using a graph theoretic framework. The necessary conditions appear in the form of a two-point boundary value problem and this is solved using a multiple shooting method. The results are then validated through numerical experiments for various cases.
\end{abstract}

\section{INTRODUCTION}

Multi-agent robotic systems find wide applications in areas such as autonomous vehicle fleet, surveillance, rescue operations etc [1] - [2]. In a system involving a large number of agents, there are always naturally occurring constraints on the communication capabilities between all the agents and the central controller, in terms of bandwidth and size of data packets to be transmitted or received. One solution to this is by employing time multiplexing of control, since this reduces the bandwidth requirement to that for communication only between one agent and the central controller.

A time multiplexed control system is a dynamical system having $M$ agents controlled by a central processing unit that can transmit a control signal at a given time to at most one among $M$ agents via a transmission channel. Here, $M$ agents work together as a joint system and are controlled through a single processor. The multiplexer selects the appropriate input signal and sends it to one of the plants. A schematic is shown in Figure 1. A scheduling of the selection is to be done in order to achieve optimized operation of the system for a specified cost function. One of the initial attempts at employing optimal control ideas in the time multiplexing problem is found in [3], and the results are illustrated in an orientation manoeuver of an ensemble of satellites.

A broad perspective on the development in multi-agent control systems is found in [4]. The survey paper illustrates

The authors thank the support of the Indo-French Centre for the Promotion of Advanced Research (IFCPRA).

A part of the second author's work was supported by a MATRICS fellowship from the Department of Science and Technology, India.

${ }^{1}$ The authors are with Systems Control Engineering, IIT Bombay, Powai, Mumbai 400076, India.

Emails: \{harsh.oza, banavar, srikant.sukumar\}

ditb.ac.in

2 The author is with Universite de Lorraine, CNRS, CRAN, F-54000 Nancy, France.

Email: constantin.morarescueuniv-lorraine.fr the work done on sensing capabilities and position or displacement based control. Apart from this, the literature covers various other aspects such as consensus algorithms, cyclic pursuit, graph-based and leader-follower strategies, etc. [5][8]. The protocols are dependent upon the availability of individual controller for each agent. In systems involving a large number of agents it is not feasible to deploy a large number of dedicated controllers or ensure full information of each agent to every other agent in the system. In order to solve this problem in [9] the authors propose a network based information multiplexing rules, which eliminates the need for global information. Yet, this method requires large number of controllers to process and implement decentralised control scheme. Existing literature in time multiplexed control cover periodic and aperiodic type of resource scheduling problems. A sparse-networked optimal scheduling problem is addressed in [10], where it is posed as a Mayer problem in continuous time. Control and scheduling strategy is jointly optimized by dynamic programming of a discrete time networked control system in [11]. An online scheduling algorithm using Mixed Integer Quadratic Programming is presented in [12]. This method is applicable for continuous and linear time invariant systems, where the ultimate objective is to provide a stabilizing control law. Model predictive control is used in [13] to pre-plan resource sharing for each agent. Only a few methods emphasize on optimality of a cost function while using time multiplexing [3], [10].

The Pontryagin maximum principle (PMP) [14] provides a set of necessary conditions on the control actions to ensure optimality. The extension to discrete time with state and control constraints is found in [15]. [16] extends the discrete PMP version to matrix Lie groups, while [17] incorporates frequency constraints into the problem. In this article, we address the problem of achieving formation under a time multiplexing constraint and with a measure of performance. We use the discrete PMP to arrive at necessary conditions of optimality and give the condition for rendezvous of agents under time multiplexing constraints. The two point boundary value problem arising from the necessary conditions is solved numerically via multiple shooting method based on Newton's root finding algorithm [18]. The rest of the paper is organized as following. The problem is presented via a mathematical statement in §II. Necessary conditions are given §III, following which numerical experiments are presented in §IV. Finally, in $\S \mathrm{V}$ concluding remarks are made. 


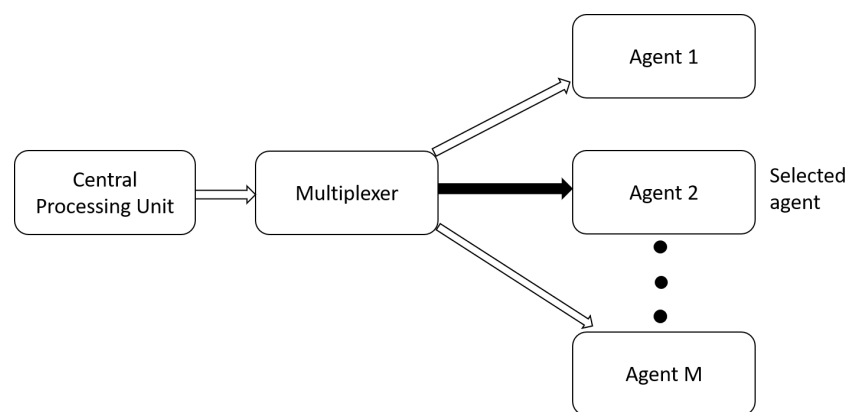

Fig. 1: A schematic representation of the time multiplexing task, where one agent is selected through the multiplexer and command is given to only that agent from the central processing unit. Rest of the agents evolve without any input.

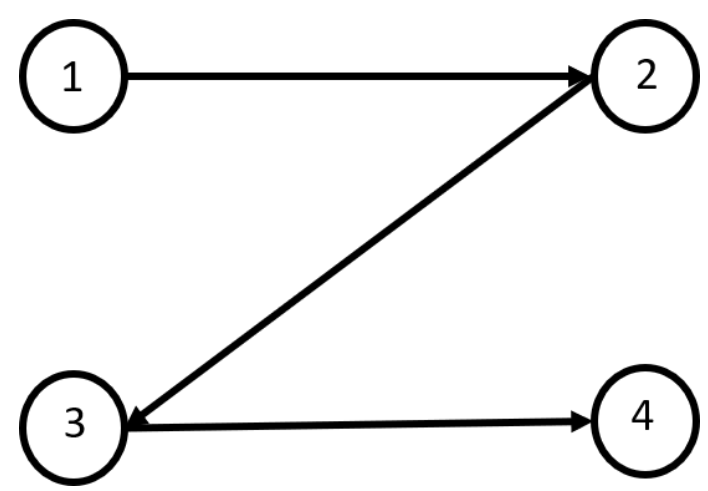

Fig. 2: An example directed graph. The direction indicates that the node at the origin of the arrow has the relative state information of its neighbours.

\section{PROBLEM FORMULATION}

\section{A. Preliminaries}

Theoretical issues in multi-agent systems have been widely tackled using graph models [2]. In this framework, the nodes of graphs represent the agents of the system and the edges of the graph represent the information availability among the agents. A graph $\mathcal{G}$ is represented by a pair $(\mathcal{V}, \mathcal{E})$, where $\mathcal{V}$ represents the set of nodes and $\mathcal{E}$ represents the set of edges. Two nodes $i$ and $j$ are said to be connected if there exists an edge between them. A directed graph is when the edges are directed from one node to another. The direction refers to the flow of information. The set of neighbors of an agent $i$ is given as $\mathcal{N}_{i}:=\{j \in \mathcal{V} \mid$ edge is directed from $\mathrm{i}$ to $\mathrm{j}\} .\left|\mathcal{N}_{i}\right|$ represent the cardinality of the set. The Laplacian matrix $\mathcal{L}:=\left[l_{i j}\right]$ of the directed graph is given by the following,

$$
l_{i j}= \begin{cases}\left|\mathcal{N}_{i}\right| & \text { if } i=j \\ -1 & \text { if } i \neq j \text { and } j \in \mathcal{N}_{i} \\ 0 & \text { otherwise }\end{cases}
$$

\section{B. Problem Setup}

We consider a system of $\mathrm{M}$ agents using time multiplexed control, where at any time instant only one agent receives a control input. Our study focusses on agents which are double integrators

$$
\ddot{x}^{(i)}=u^{(i)} \quad i \in[M]^{*}
$$

We set $[M]:=\{0,1,2, \cdots, M\}$ and $[M]^{*}:=[M] \backslash\{0\}$. $x^{(i)} \in \mathbb{R}^{m}$ and $u^{(i)} \in \mathbb{R}^{m}$ represent the position and control of $i^{\text {th }}$ agent respectively. The system in discrete time is represented by,

$$
\begin{aligned}
& x_{k+1}^{(i)}=x_{k}^{(i)}+h v_{k}^{(i)} \\
& v_{k+1}^{(i)}=v_{k}^{(i)}+h u_{k}^{(i)}
\end{aligned}
$$

where

i) $x_{k}^{(i)} \in \mathbb{R}^{m}$ and $v_{k}^{(i)} \in \mathbb{R}^{m}$ are the position and velocity of the $i^{t h}$ agent at $k^{t h}$ instant of time, where $k \in[N-1]$ and $i \in[M]^{*}$.

ii) $u_{k}^{(i)}$ is the admissible control input to the $i^{\text {th }}$ agent.

iii) $h$ is the discretization step.

The agents are assumed to have information on each of their neighbours' states. We use a graph theoretic framework to depict the information sharing topology. The associated graph is $\mathcal{G}$ and graph Laplacian matrix is $\mathcal{L}$ and the new states are

$$
\left(\begin{array}{c}
z_{k}^{(1)} \\
z_{k}^{(2)} \\
\vdots \\
z_{k}^{(M)}
\end{array}\right)=\mathcal{L}\left(\begin{array}{c}
x_{k}^{(1)} \\
x_{k}^{(2)} \\
\vdots \\
x_{k}^{(M)}
\end{array}\right) \text { and }\left(\begin{array}{c}
y_{k}^{(1)} \\
y_{k}^{(2)} \\
\vdots \\
y_{k}^{(M)}
\end{array}\right)=\mathcal{L}\left(\begin{array}{c}
v_{k}^{(1)} \\
v_{k}^{(2)} \\
\vdots \\
v_{k}^{(M)}
\end{array}\right)
$$

where, $z_{k}^{(i)}$ and $y_{k}^{(i)}$ denote the sum of relative positions and velocities of $i^{\text {th }}$ agent with respect to its neighbours, respectively. Collecting all specific states of each agent, we denote $Z_{k}:=\left(z_{k}^{(1)}, z_{k}^{(2)}, \cdots, z_{k}^{(M)}\right)^{T}$ as the joint position variable of all agents, $Y_{k}:=\left(y_{k}^{(1)}, y_{k}^{(2)}, \cdots, y_{k}^{(M)}\right)^{T}$ as the joint velocity variable of all agents and $U_{k}:=\left(u_{k}^{(1)}, u_{k}^{(2)}, \cdots, u_{k}^{(M)}\right)^{T}$ as the joint control input of all agents. In terms of the new variables, the dynamics are

$$
\begin{array}{r}
Z_{k+1}=Z_{k}+h Y_{k} \\
Y_{k+1}=Y_{k}+h\left(\mathcal{L} U_{k}\right)
\end{array}
$$

The cost is defined as

$$
J\left(Z_{k}, Y_{k}, U_{k}\right)=\phi_{N}\left(Z_{N}, Y_{N}\right)+\sum_{k=0}^{N-1} \phi_{k}\left(Z_{k}, Y_{k}, U_{k}\right)
$$

where, $\phi_{k}\left(Z_{k}, Y_{k}, U_{k}\right)$ is a map which gives the cost per stage and $\phi_{N}\left(Z_{N}, Y_{N}\right)$ is a map which gives terminal stage cost. We now formally state the optimal control problem.

\section{Problem Statement:}

$$
\min _{U_{k}} J\left(Z_{k}, Y_{k}, U_{k}\right)
$$


subject to

$$
\text { constraints } \begin{cases}\text { dynamics }(5) & \\ Z_{0}=Z_{\text {in }} & \\ Y_{0}=Y_{\text {in }} & \\ g\left(U_{k}\right) \leq 0 & \forall k \in[N-1] \\ \phi_{N}\left(Z_{N}, Y_{N}\right): \text { specifies } & \\ U_{k} \in \mathbb{U} & \forall k \in[N-1]\end{cases}
$$

where

i) $g\left(U_{k}\right) \leq 0$ is a map which restricts the magnitude of control input;

ii) $Z_{\text {in }} \in \mathbb{R}^{m} \times \cdots \times \mathbb{R}^{m}$ ( $M$ times) refers to the fixed initial positions of all agents;

iii) $Y_{\text {in }} \in \mathbb{R}^{m} \times \cdots \times \mathbb{R}^{m}$ ( $M$ times) refers to the fixed initial velocities of all agents;

The time multiplexing constraint on set of controls is realized by defining the admissible control set in following manner.

$$
\mathbb{U}:=\left\{\left(\begin{array}{c}
r \\
0 \\
\vdots \\
0
\end{array}\right),\left(\begin{array}{c}
0 \\
r \\
\vdots \\
0
\end{array}\right), \cdots,\left(\begin{array}{c}
0 \\
0 \\
\vdots \\
r
\end{array}\right) \mid r \in[-1,1]\right\}
$$

This definition allows us to provide control input to only one agent at a given time-step and the rest of the agents evolve without any control input during that stage.

\section{METHODOLOGY}

\section{A. Transformation of admissible control set into a convex set}

The admissible time multiplexing constraint defined in (9) is a star-shaped set. To impart convexity to incorporate this constraint, we perform the following mathematical jugglery. We define an auxiliary state $w$ as follows:

$$
w_{k+1}=w_{k}+\psi\left(U_{k}\right) ; \quad w_{0}=(0,0)^{T}
$$

where the map,

$$
\begin{array}{r}
\psi\left(U_{k}\right):=\sum_{i=0}^{M-1} \sum_{j=1}^{M}\left(\left\|u^{(i)}\right\|^{2}\left\|u^{(i)}\right\|^{2}\left(\begin{array}{l}
1 \\
1
\end{array}\right)\right. \\
\left.+u^{(i)} \odot u^{(j)}\left(\begin{array}{c}
1 \\
-1
\end{array}\right)\right)
\end{array}
$$

and

$$
\begin{aligned}
u^{(i)} \odot u^{(j)} & :=\left\langle W u^{(i)},\left(u^{(j) T}, u^{(j) T}, \ldots, u^{(j) T}(\mathrm{~m} \text { times })\right)^{T}\right\rangle \\
W_{i j} & := \begin{cases}1 & \text { if }(j-1) m+1 \leq i \leq j m \\
0 & \text { else }\end{cases}
\end{aligned}
$$

The proof of equivalence between the set $\mathbb{U}$ and the map $\psi$ is given in [3].

Theorem: Let $\left(\tilde{U}_{k}\right)_{k=0}^{N-1}$ be an optimal control trajectory for
(7) and $\left(\tilde{Z}_{k}\right)_{k=0}^{N},\left(\tilde{Y}_{k}\right)_{k=0}^{N}$ and $\left(\tilde{w}_{k}\right)_{k=0}^{N}$ be the optimal state trajectory. Define the Hamiltonian,

$$
\begin{array}{r}
\mathcal{H}_{k}\left(\lambda^{0}, \lambda_{k}, \alpha_{k}, Z_{k}, Y_{k}, U_{k}\right):=\lambda^{0} \phi_{k}\left(Z_{k}, Y_{k}, w_{k}, U_{k}\right)+ \\
\left\langle\lambda_{k}, F_{k}\left(Z_{k}, Y_{k}, U_{k}\right)\right\rangle+\left\langle\alpha_{k}, w_{k}\right\rangle
\end{array}
$$

where, $\lambda_{k}=\left(\begin{array}{c}\lambda_{k}^{1} \\ \lambda_{k}^{2}\end{array}\right)$ and

$F_{k}\left(Z_{k}, Y_{k}, U_{k}\right):=\left(\begin{array}{c}Z_{k}+h Y_{k} \\ Y_{k}+h\left(\mathcal{L} U_{k}\right)\end{array}\right)$ Then there exist corresponding $\tilde{\lambda^{0}} \in\{-1,0\}, \tilde{\lambda_{k}} \in \mathbb{R}^{2 m}$ and $\tilde{\alpha_{k}} \in \mathbb{R}^{2}$ such that the following necessary conditions hold. The necessary conditions for optimal control in discrete time can be arrived at using [15].

(i) the non triviality condition: adjoint variables $\tilde{\lambda^{0}}, \tilde{\lambda_{k}}$ and $\tilde{\alpha_{k}}$ do not vanish simultaneously.

(ii) state:

$$
\begin{aligned}
\tilde{Z}_{k+1} & =\tilde{Z}_{k}+h \tilde{Y}_{k} \\
\tilde{Y}_{k+1} & =\tilde{Y}_{k}+h\left(\mathcal{L} \tilde{U}_{k}\right) \\
\tilde{w}_{k} & =\tilde{w}_{k}+\psi\left(\tilde{U}_{k}\right)
\end{aligned}
$$

adjoint:

$$
\begin{aligned}
\tilde{\lambda}_{k-1}^{1} & =\lambda^{0} \frac{\partial \phi_{k}\left(Z_{k}, Y_{k}, U_{k}\right)}{\partial Z_{k}}+\tilde{\lambda}_{k}^{1} \\
\tilde{\lambda}_{k-1}^{2} & =\lambda^{0} \frac{\partial \phi_{k}\left(Z_{k}, Y_{k}, U_{k}\right)}{\partial Y_{k}}+h \tilde{\lambda}_{k}^{1}+\tilde{\lambda}_{k}^{2} \\
\tilde{\alpha}_{k-1} & =\tilde{\alpha}_{k} \\
\forall k \in[N-1] &
\end{aligned}
$$

(iii) the Hamiltonian non-positivity:

$$
\begin{array}{r}
\left(\lambda^{0} \frac{\partial \phi_{k}\left(Z_{k}, Y_{k}, U_{k}\right)}{\partial U_{k}}+\tilde{\lambda}_{k}^{2}(\mathcal{L}) U_{k}\right) \delta U \\
\leq 0 \\
\tilde{U}+\delta U \in \mathbb{U} \\
\forall k \in[N-1]
\end{array}
$$

\section{B. Condition for achieving the formation}

As we have assumed a graph structure where agents have relative information of other agents, it is natural to define the formation as a function of relative state. We embed the formation objective in the end stage constraint given by $\phi_{N}\left(Z_{N}, Y_{N}\right)$. For example, if our objective is rendezvous, $\phi_{N}\left(Z_{N}, Y_{N}\right):=Z_{N}=0$. This is utilized in defining the boundary conditions $(\mathcal{B})$, defined later.

\section{Multiple Shooting Method}

Following the necessary condition, solution of the Two Point Boundary Value Problem(TPBVP) is found using numerical technique of multiple shooting. In multiple shooting method, the time horizon is divided in sub interval and a guess of every unknown state at each stage is taken. Using the dynamics of the system each guess is corrected iteratively till a tolerance bound is achieved. 
We have $Z_{k}, Y_{k}, w_{k}$ and $\lambda_{k}$ as unknown states for all $k \in$ $[N]$ and the states are driven by dynamics (13) and (14). We define our guess vector in following way.

$$
\begin{array}{r}
X:=\left(Z_{k}, Y_{k}, w_{k}, \lambda_{k}, \alpha_{k}\right)^{T} \\
\forall k \in[N]
\end{array}
$$

The boundary conditions are as following.

$$
\begin{aligned}
\mathcal{B}: & Z_{0}=Z_{\text {in }} \\
& Y_{0}=Y_{\text {in }} ; \\
& \phi_{N}\left(Q_{N}\right)=\text { user defined; } \\
& w_{N}=(0,0)^{T}
\end{aligned}
$$

The guess variables are fed to the dynamics of state and adjoint given in (13) and (14) respectively with each guess variable acting as an initial condition. The guess is true only if the solution to $k^{t h}$ iterate is equal to the guess of $(k+1)^{t h}$ variable. this is called matching conditions. All matching conditions are combined and represented as $F(X)=0$, where

$$
F(X):=\left(\begin{array}{c}
Z_{k+1}-\left(Z_{k}+h Y_{k}\right) \\
Y_{k+1}-\left(Y_{k}+h\left(\mathcal{L} U_{k}\right)\right) \\
w_{k+1}-\left(w_{k}+\psi\left(U_{k}\right)\right) \\
\tilde{\lambda}_{k-1}^{1}-\left(\lambda^{0} \frac{\partial \phi_{k}\left(Z_{k}, Y_{k}, U_{k}\right)}{\partial Z_{k}}+\tilde{\lambda}_{k}^{1}\right) \\
\tilde{\lambda}_{k-1}^{2}-\left(\lambda^{0} \frac{\partial \phi_{k}\left(Z_{k}, Y_{k}, U_{k}\right)}{\partial Y_{k}}+h \tilde{\lambda}_{k}^{1}+\tilde{\lambda}_{k}^{2}\right) \\
\alpha_{k-1}-\left(\frac{\partial \mathcal{H}_{k}\left(\lambda^{0}, \lambda_{k}, \alpha_{k}, Z_{k}, Y_{k}, w_{k}, U_{k}\right)}{\partial w_{k}}\right) \\
\mathcal{B} \quad \forall k \in[N-1]
\end{array}\right)
$$

The solution of the problem (18) can be found using Newton's root finding algorithm. First, we choose an appropriate error bound $\epsilon$, which makes a terminating condition for our algorithm. Till $\|F(X)\|_{\infty}<\epsilon$ following steps are repeated.

(1) Calculate $\Delta X$ using the $\partial_{X} F(X) \Delta X=-F(X)$, where $\partial_{X} F(X)$ is the Jacobain of function $F(X)$ with respect to $X$.

(2) Update the value of $X=X+\Delta X$

(3) Calculate $\|F(X)\|_{\infty}$

Once the error is less than the tolerance bound, we conclude that the trajectories are optimal. Remark: The Newton's method converges only when the initial guess is reasonably close to the solution, and further, if there exists a solution to the problem. Hence, if the optimal control problem under the specific set of constraints is not feasible Newton's method will not yield a solution.

\section{NUMERICAL EXPERIMENTS}

We verify our theory with numerical experiments on four double integrator agents evolving on $\mathbb{R}^{2}$ with the graph topology shown in Fig. 2. We optimize the control input with respect to the cost $\phi_{k}\left(Z_{k}, Y_{k}, U_{k}\right):=\sum_{k=0}^{N}\left\|U_{(k)}\right\|_{2}^{2}$. Additionally, we impose constraints on magnitude of control

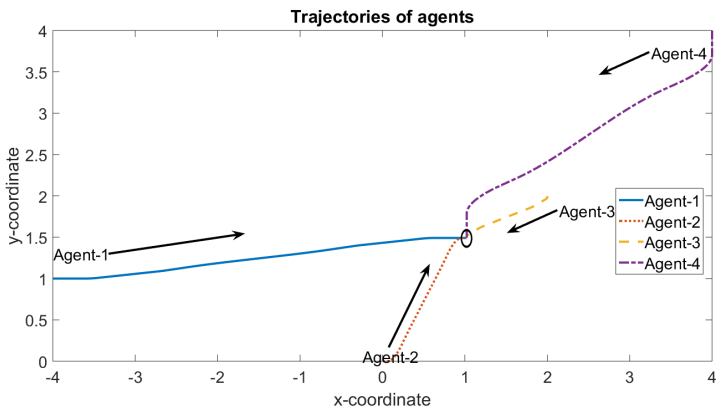

Fig. 3: Trajectory of the agents reaching to a point in $\mathrm{N}$ discrete time stages

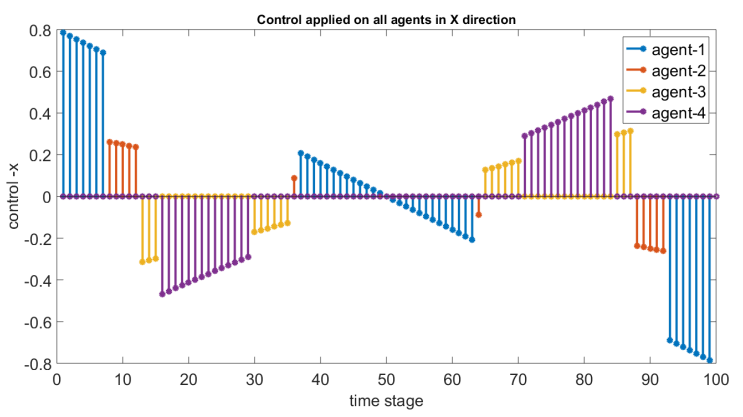

Fig. 4: X-coordinate of control

as $g\left(U_{k}\right):=\left\|U_{k}\right\|_{\infty}-1 \leq 0$, which is natural in practical cases. For the computation purpose we have taken the discertization step size $h=0.1$ and the number of discrete time stages as $N=100$. We test our proposed solution technique in two different scenarios: (a) A rendezvous problem (RP) and (b) a formation problem (FP).

\section{A. Case 1: Rendezvous problem}

In this case, all the agents have to reach a common position. All the agents start from different positions. The final time conditions are given as following.

$$
\phi_{N}\left(Z_{N}, Y_{N}\right):=Z_{N}=0
$$

Fig. 4 and Fig. 5 show the control inputs applied to all the agents and it is clear that when one agent is receiving input the others are not. and they reach an identical point, as shown in Fig. 3. The energy utilized in this case is 12.58 units.

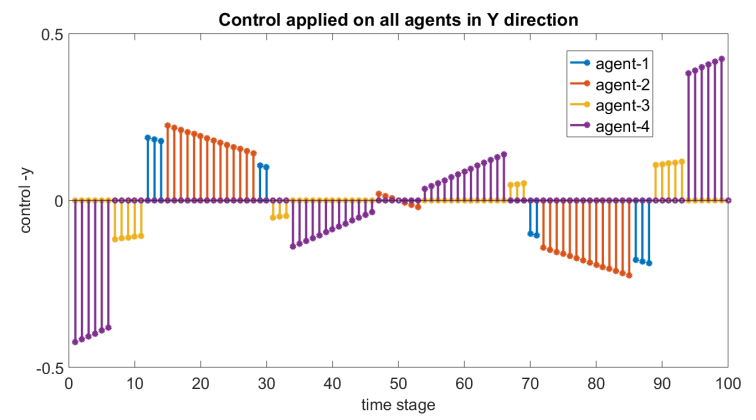

Fig. 5: Y-coordinate of control 


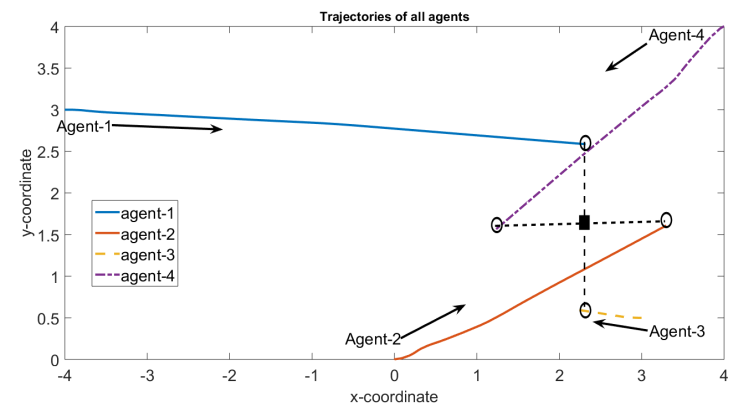

Fig. 6: Trajectory of the agents forming a '+' shape

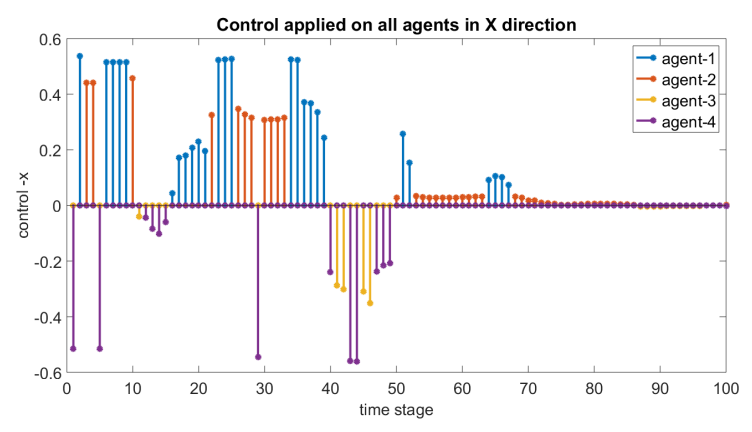

Fig. 7: X-coordinate of control

\section{B. Case 2: Formation problem}

In this case, the agents are required to achieve a specified formation. The agents make a '+' shape. This is codified by,

$$
\phi_{N}\left(Z_{N}\right):=Z_{N}-d=0
$$

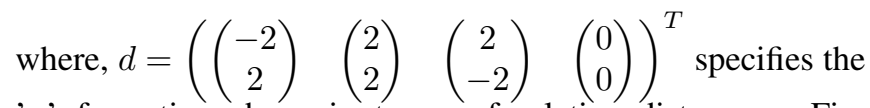
' + ' formation shape in terms of relative distances. Fig.

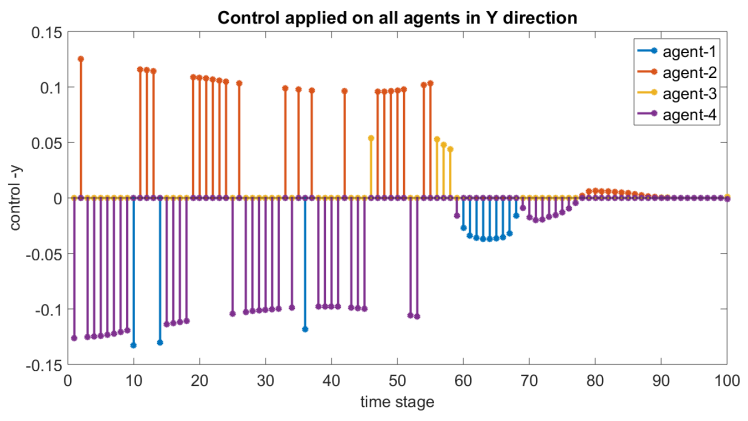

Fig. 8: Y-coordinate of control

6 shows that all agents start from given initial states and achieve the desired ' + ' shape. Similar to case I, multiplexed control can be observed in Fig. 7 and Fig. 8.

\section{CONCLUSIONS}

We have presented some early findings of our investigations into time multiplexing being employed for objectives in multiagent problems using the framework of constrained discrete optimal control. In the future we hope to drastically increase the number of agents, and make more conclusive assertions on the advantages and disadvantages of time multiplexing from various perspectives. We are also working on the numerical aspects of solving the TPBVP using alternate algorithms.

\section{REFERENCES}

[1] Francesco Bullo, Jorge Cortes, and Sonia Martinez. Distributed control of robotic networks: a mathematical approach to motion coordination algorithms. Princeton University Press, 2009.

[2] Mehran Mesbahi and Magnus Egerstedt. Graph theoretic methods in multiagent networks. Princeton University Press, 2010.

[3] Chinmay Maheshwari, Sukumar Srikant, and Debasish Chatterjee. On optimal multiplexing of an ensemble of discrete-time constrained control systems on matrix lie groups. arXiv preprint arXiv:1902.08404, 2019.

[4] Kwang-Kyo Oh, Myoung-Chul Park, and Hyo-Sung Ahn. A survey of multi-agent formation control. Automatica, 53:424-440, 2015.

[5] Reza Olfati-Saber and Richard M Murray. Consensus problems in networks of agents with switching topology and time-delays. IEEE Transactions on automatic control, 49(9):1520-1533, 2004.

[6] Joshua A Marshall, Mireille E Broucke, and Bruce A Francis. Formations of vehicles in cyclic pursuit. IEEE Transactions on automatic control, 49(11):1963-1974, 2004.

[7] Zhiyun Lin, Lili Wang, Zhimin Han, and Minyue Fu. Distributed formation control of multi-agent systems using complex laplacian. IEEE Transactions on Automatic Control, 59(7):1765-1777, 2014.

[8] Luca Consolini, Fabio Morbidi, Domenico Prattichizzo, and Mario Tosques. Leader-follower formation control of nonholonomic mobile robots with input constraints. Automatica, 44(5):1343-1349, 2008.

[9] Dzung Tran, Tansel Yucelen, and Eduardo L Pasiliao. Formation control with multiplex information networks. IEEE Transactions on Control Systems Technology, 2018.

[10] Yogesh Kumar, Sukumar Srikant, and Debasish Chatterjee. Optimal multiplexing of sparse controllers for linear systems. Automatica, 106:134-142, 2019.

[11] Daniel Görges, Michal Izák, and Steven Liu. Optimal control and scheduling of networked control systems. In Proceedings of the $48 \mathrm{~h}$ IEEE Conference on Decision and Control (CDC) held jointly with 2009 28th Chinese Control Conference, pages 5839-5844. IEEE, 2009.

[12] M El Mongi Ben Gaid, Arben Cela, and Yskandar Hamam. Optimal integrated control and scheduling of systems with communication constraints. In Proceedings of the 44th IEEE Conference on Decision and Control, pages 854-859. IEEE, 2005.

[13] Kazumune Hashimoto, Shuichi Adachi, and Dimos V Dimarogonas. A collision-free communication scheduling for nonlinear model predictive control. IFAC-PapersOnLine, 50(1):8939-8944, 2017.

[14] LS Pontryagin and VG Boltyanskii. R. v. gamkrelidze, and e. F. Mishchenko, "The Mathematical Theory of Optimal Processes," translated by K. N. Trirogoff, Interscience. New York, 1962.

[15] Vladimir Grigor'evich Bolt'yanskii. The method of tents in the theory of extremal problems. Russian Mathematical Surveys, 30(3):1, 1975.

[16] Karmvir Singh Phogat, Debasish Chatterjee, and Ravi N Banavar. A discrete-time pontryagin maximum principle on matrix lie groups. Automatica, 97:376-391, 2018.

[17] Shruti Kotpalliwar, Pradyumna Paruchuri, Karmvir Singh Phogat, Debasish Chatterjee, and Ravi Banavar. A frequency-constrained geometric pontryagin maximum principle on matrix lie groups. In 2018 IEEE Conference on Decision and Control (CDC), pages 4348. IEEE, 2018.

[18] Hans Georg Bock and Karl-Josef Plitt. A multiple shooting algorithm for direct solution of optimal control problems. IFAC Proceedings Volumes, 17(2):1603-1608, 1984. 